\title{
Rechtspopulismus in Betrieben und Regionen: eine Herkulesaufgabe für die Betriebs- und Gewerkschaftspolitik
}

\author{
CLAUDIA PETER, MICHAEL BRECHT
}

Dass es diskriminierende, ausgrenzende oder rassistische Vorfälle gibt, ist in den Betrieben unserer Region nicht grundsätzlich neu. IG Metall und Betriebsräte haben sich hierzu positioniert. Mit der Landtagswahl 2016 gab es jedoch eine offene Zäsur: Im Landkreis Rastatt entfielen $17,6 \%$ der Stimmen auf die AfD, in einigen Wahlkreisen der Region sogar $30 \%$. Bei der Bundestagswahl 2017 lag der regionale Stimmenanteil der AfD bei 11,5\%. Und seit dem Frühjahr 2018 sitzen drei Betriebsratsmitglieder vom extrem rechten „Zentrum Automobil“ im 35-köpfigen Betriebsrat bei Mercedes-Benz in Rastatt.

Folgt man den gängigen Erklärungen zum Erstarken rechtspopulistischer Einstellungen - soziale Benachteiligung, Zukunftsangst, Chancenlosigkeit, das Gefühl, „abgehängt“ zu sein -, so muss die skizzierte Entwicklung überraschen: Die Region ist wirtschaftlich sehr stark. Knapp 30\% der sozialversicherungspflichtigen Beschäftigten sind mit der Autoherstellung bzw. der Produktion von Zulieferteilen beschäftigt. Der Median der Entgelte liegt bei $3600 €$ (2015), die Arbeitslosenquote bei nur 3,2\%. Die Arbeitsplätze in den Betrieben sind relativ sicher. Betriebe und Wirtschaft sind auf Wachstum ausgerichtet. Es handelt sich also um eine Region mit hoher Stabilität; auch bezüglich der Zukunftsaussichten. Ansonsten ergibt sich aus der politischen Einstellung großer Teile der Bevölkerung kein außergewöhnliches Reservoir an Protestwählern - im Gegenteil: Mehrheitlich wird politisch konservativ gewählt.

Die gewerkschaftlichen Organisationsgrade in den von der IG Metall betreuten Betrieben sind recht hoch. Die beiden hier ansässigen Großbetriebe von Daimler haben Organisationsgrade über 70 bzw. über $80 \%$, die mittelständischen Betriebe oftmals ebenfalls weit über $50 \%$ bis zu 80\%. Die IG Metall ist gesellschaftlich und politisch eine feste Größe in der Region und erfreut sich hoher Akzeptanz, was sich auch in der Mitgliedschaft zeigt: Die IG Metall Gaggenau hat derzeit rund 24400 Mitglieder, davon 14400 aus den Betrieben. Stabil sind auch die Strukturen betrieblicher Interessenvertretung im Merce-
des-Benz-Werk Gaggenau: Bei knapp 7000 Beschäftigten gibt es einen 33-köpfigen Betriebsrat und eine funktionierende gewerkschaftliche Vertrauensleutearbeit.

\section{Was tun?}

Natürlich haben sich IG Metall, Betriebsräte und gewerkschaftliche Vertrauensleute ausführlich mit den Ursachen für das Erstarken der AfD und ihrer „betrieblichen Ableger" beschäftigt und nach Handlungsmöglichkeiten gesucht, um ihm ein solidarisches Verständnis vom Umgang miteinander - in Betrieb und Gesellschaft - entgegenzusetzen. Zu unseren Erfahrungen zählt: Es gibt kein Patentrezept, um mit Rechtspopulismus in den Betrieben und in der Öffentlichkeit umzugehen. Und bei Aktivitäten stellt sich hinterher stets die Frage: Hat das wirklich genutzt?

So stellt sich für die IG Metall und betriebliche Interessenvertretungen anhaltend die Aufgabe, immer wieder nach Möglichkeiten zu suchen und keine Gelegenheit auszulassen, sich zu positionieren: durch aktives Engagement für eine offene, demokratische und respektvolle Arbeitswelt und Gesellschaft, durch das Vorleben gewerkschaftlicher und humanistischer Werte und die Ausrichtung der (Gewerkschafts-)Politik daran. Dabei wird sich die IG Metall inhaltlich nicht von den Rechten treiben lassen. Das aktive Handeln für gute Arbeit und eine offene, gerechte Gesellschaft stehen im Mittelpunkt gewerkschaftlichen Handelns. Daran sind IG Metall und betriebliche Interessenvertretungen zu messen. Über den "richtigen Weg“ wird es natürlich immer wieder Diskussionen geben. Fakt aber ist: Sich klar zu positionieren ist ein zentraler Punkt in der (betrieblichen) Öffentlichkeitsarbeit - von der Diskussion in Pausenräumen über Betriebsversammlungen bis hin zu öffentlichen Veranstaltungen.

Sehr hilfreich sind gemeinsame Positionierungen von Arbeitgebern und Betriebsräten. Das wird beim „Benz“ in Gaggenau gelebt: Gegen Rechtspopulismus stehen Betriebsrat und Werkleitung Seite an Seite. Aber wie schaffen es Gewerkschafterinnen und Gewerkschafter, an die Köpfe 
der Menschen heranzukommen? Kann das Vorbild eines respektvollen Umgangs und Denkens ausgrenzendes Denken und Handeln beeinflussen?

Insgesamt ist das (Schulungs-)Material, das es von der IG Metall und anderen Organisationen gibt, hilfreich und hervorragend. Unsere Erfahrung zeigt aber auch: Material allein nützt wenig, wenn wir es nicht schaffen, dass sich Beschäftigte aktiv für demokratische Werte einsetzen. Deswegen hat die IG Metall Gaggenau im Herbst 2016 eine Wertekampagne gestartet, mit der wir sowohl in die Betriebsöffentlichkeit als auch in die gesellschaftliche Öffentlichkeit hineinwirken wollen.

\section{Wertekampagne}

Ausgehend von der Satzung der IG Metall wurde bei einer Delegiertenversammlung der Startpunkt für diese Kampagne gelegt. Die Wertekampagne soll das ausdrückliche und wahrnehmbare Bekenntnis für gewerkschaftlichen Werte sein: Sie soll

- eine positive Diskussion über gewerkschaftliche Werte bewirken,

- eine eindeutige Abgrenzung zu rechtspopulistischen Werten verdeutlichen,

- Anstöße für ein den gewerkschaftlichen Werten entsprechendes Gesellschaftsbild geben,

- Denken und Handeln entsprechend beeinflussen.

In der Diskussion wurde deutlich, wie wichtig es ist, sich für etwas einzusetzen und zu diskutieren, statt Gegen-Positionen zu beziehen und damit immer in der Abwehrhaltung zu verharren. Sich für Werte und ein entsprechendes Gesellschaftsbild stark zu machen ist zum einen eine positive Denk- und Herangehensweise, ein positiver Handlungsansatz; zum anderen verdeutlicht es die klare Abgrenzung zu rechtspopulistischem Denken und Handeln.

In den Wochen und Monaten nach der Delegiertenversammlung wurden verschiedene Aktivitäten gestartet:

- ein Wertelogo wurde kreiert, das seitdem im Einsatz ist;

- betrieblich wichtige und bekannte Persönlichkeiten "zeigen Gesicht“, sie unterstützen das Anliegen der Wertekampagne, worüber in den Betrieben an den „Schwarzen Brettern“ und auf der Homepage der IG Metall Gaggenau informiert wird;

- in Betriebsversammlungen, betrieblichen Gremiensitzungen und Vertrauensleute-Vollversammlungen werden die Werte thematisiert und diskutiert;

- im Sinne eines breiteren öffentlichkeitswirksamen Wirkens werden auch die Veranstaltungen und Reden zum 1. Mai für die Wertekampagne genutzt;

- gleichfalls im Sinne öffentlichkeitswirksamer Aktivitäten sucht die IG Metall Gaggenau weit über den „Dunstkreis“ gewerkschaftlicher Funktionsträger und gewerkschaftlich Aktiver hinaus die Unterstützung prominenter Repräsentanten demokratischer Werte. So konnte der Bundestagspräsident Norbert Lammert für eine Veranstaltung der IG Metall Gaggenau als Unterstützer gewonnen werden;

- und die IG Metall hat Kandidatinnen und Kandidaten der Bundestagswahl 2017 eingeladen, um mit ihnen deren Wahlprogramm im Lichte gewerkschaftlicher Ziele und Werte kritisch zu diskutieren. Zu diesen Veranstaltungen wurden kurze Videoclips produziert und online gestellt.

Es lohnt sich, im Folgenden ein kurzes Schlaglicht auf die verschiedenen Aktivitäten und Veranstaltungen zu werfen, nicht nur um errungene Erfolge aufzuzeigen, sondern auch um Schwierigkeiten und Bruchstellen zu verdeutlichen.

\section{Der Betrieb als Handlungsfeld}

Die Wahlanalysen nach der Landtagswahl 2016 zeigten, dass der Anteil der AfD-Wählerinnen und -Wähler unter Gewerkschaftsmitgliedern höher ist als im Durchschnitt der abgegebenen Stimmen. In der Feinanalyse wurde deutlich, dass fast ein Fünftel der Gewerkschaftsmitglieder in Baden-Württemberg die AfD gewählt hat. Bei den Diskussionen zu unserer Wertekampagne war schnell klar: Wir wollen die AfD-nahen Wählerinnen und Wähler nicht für ihr Wahlverhalten beschimpfen, allerdings wollen wir respektlose und ausgrenzende Parolen und Äußerungen auch nicht im Raum stehen lassen. Deswegen gilt die Devise: Wir wollen uns positionieren, aber nicht gegen die Menschen, sondern für gewerkschaftliche Werte. Was wir vermitteln wollen, wurde innerhalb der IG Metall-Geschäftsstelle gemeinsam abgestimmt, sodass alle Gewerkschaftssekretärinnen und -sekretäre mit denselben Botschaften in die Betriebe gehen.

Und trotzdem zeigen die Erfahrungen in der betrieblichen Realität Unwägbarkeiten. So berichten beispielsweise "gestandene“ aktive Gewerkschafter, dass sie nicht mehr gerne in die „Raucherecke“ im Betrieb gehen, weil sie dort bewusst mit fremdenfeindlichen Sprüchen provoziert werden. Ein anderer Kollege berichtet, dass infolge der Zusage von Daimler, zusätzliche Ausbildungsplätze für junge Flüchtlinge zu schaffen, eine Neiddebatte unter dem Tenor entbrannt sei: „Da kriegt ein Flüchtling einen Ausbildungsplatz beim Benz - und mein Sohn nicht“.

Es gehört schon Rückgrat dazu, in solchen Situationen immer wieder für respektvolle Werte einzustehen. Und es ist wichtig, was in der betrieblichen Öffentlichkeit vermittelt und wahrgenommen wird: über die „Schwarzen Bretter", in Sitzungen und (Betriebs-)Versammlungen. Aber die eigentlich schwierige Aufgabe ist die alltägliche Debatte.

Der Umgang mit rechtspopulistischen Tendenzen spielt in einem Konzern wie Daimler natürlich auch in der Arbeit des Gesamtbetriebsrats eine Rolle. Ebenso gibt es 
gemeinsame Erklärungen und Statements, die sich gegen Ausgrenzung und Fremdenfeindlichkeit positionieren, vonseiten des Vorstands und des Vorstandsvorsitzenden. Sie sind zum Glück eine Selbstverständlichkeit und müssen nicht ausdrücklich eingefordert werden.

Integration funktioniert am besten über (gemeinsame) Arbeit und Kommunikation. Um Vorbehalte gegenüber Geflüchteten abzubauen und sie stattdessen zu akzeptierten Kolleginnen und Kollegen zu machen, stellte der Konzern in mehreren Wellen einige hundert Plätze für sogenannte Brückenpraktika zur Verfügung. Das heißt, es wurden zusätzliche Ausbildungsplätze angeboten, um einerseits jungen Geflüchteten eine Perspektive zu bieten und andererseits den kollegialen Kontakt zu befördern. Diese Maßnahmen fruchten. Sie lösen zwar nicht die Integrationsprobleme insgesamt, sind aber ein wichtiger Baustein hinsichtlich gesellschaftlicher und politischer Verantwortung.

\section{Betriebsratswahl und „Zentrum Automobil“}

Im Herbst 2017 wird über Facebook gepostet, dass das „Zentrum Automobil“, eine auf Betriebsebene agierende, in der militanten Rechten verankerte Gruppe (vgl. Schroeder et al. in diesem Heft), eine zehnköpfige Betriebsgruppe im Mercedes-Benz-Werk Rastatt etabliert habe und sich für die Betriebsratswahl im März 2018 in Stellung bringe. Die Wogen schlugen schnell hoch und erste Reflexe waren, sofort eine Gegenkampagne zu starten, eventuelle IG-Metall-Mitglieder auf der Liste des „Zentrums“ auszuschließen und eine Kundgebung gegen das „Zentrum Automobil“ zu organisieren. Auch die Medien stürzten sich sofort auf dieses Thema. Zwischenzeitlich steigerte die mediale Aufmerksamkeit die Wahrnehmung und führte zu dem irrigen Eindruck, es gebe rechtspopulistische Kandidaten bei vielen Betriebsratswahlen. Zugleich war die mediale Erwartungshaltung an die IG Metall vor Ort in einigen Fällen sehr eindimensional: Warum macht die IG Metall keinen „Rabatz“ und ruft zu öffentlichkeitswirksamen Aktionen auf?

Die IG Metall Gaggenau hat sich in ihrer betrieblichen und überbetrieblichen Arbeit stattdessen - gemeinsam mit der ihr nahe stehenden „Rastatter Liste“ - an den Grundsätzen ihrer Wertekampagne orientiert und in Debatten wie auch in ihren Publikationen verdeutlicht, wofür sie in der betrieblichen Politik steht, welches die relevanten $\mathrm{Zu}$ kunftsthemen sind, wie diese aufgegriffen werden müssen und wo mit Blick auf gesellschaftliche relevante Zukunftsthemen und Werthaltungen die signifikanten Unterschiede zu den Positionen des "Zentrums Automobil“ liegen. ${ }^{1}$

Die IG Metall hat im Vorfeld der Betriebsratswahl ausdrücklich signalisiert, mit all jenen im neuen Betriebsratsgremium zusammenzuarbeiten, die gewerkschaftliche Werte teilen. In diesem Zusammenhang wurde auch sehr genau darauf geschaut, ob sich die Kandidaten im Betrieb rassistisch und/oder fremdenfeindlich betätigen. Hier- für ist mittlerweile eine hohe Sensibilität vorhanden. Zur Betriebsratswahl traten schließlich Kandidatinnen und Kandidaten von insgesamt neun Listen an. Acht dieser Listen erzielten Sitze im neukonstituierten Betriebsratsgremium. 32 der 35 Betriebsratsmitglieder arbeiten wie eine „Groß-Fraktion“ zusammen (sie sind alle Mitglieder der IG Metall). Ausgenommen sind lediglich die drei Betriebsratsmitglieder vom „Zentrum Automobil“.

\section{Flagge zeigen - auch in der gesellschaftlichen, außerbetrieblichen Öffentlichkeit}

Es ist ein Kernanliegen der IG-Metall-Wertekampagne, gewerkschaftliche und demokratische Werte nicht nur im Rahmen der gewerkschaftlichen und betrieblichen Arbeit im engeren Sinne zu vermitteln, sondern hierfür auch in der Gesellschaft insgesamt, in ihren verschiedenen Teil-Öffentlichkeiten einzustehen.

Eine Gelegenheit hierfür bot der AfD-Landesparteitag, der im Mai 2017 in Rastatt stattfand. Auch hier sind wir nicht dem ersten Reflex gefolgt, einfach eine Gegendemo zu organisieren, sondern die IG Metall Gaggenau hat sich für eine andere Vorgehensweise entschieden, um auch Menschen aus der politischen Mitte zu erreichen, die nicht unbedingt zu einer Kundgebung kommen. Unser vorrangiges Ziel war es, ein breites gesellschaftliches und politisches Bündnis zu schmieden. Hierbei war der Umstand hilfreich, dass die IG Metall Gaggenau eine hohe Akzep$\tan z$ in der örtlichen demokratischen Parteienlandschaft genießt. Unter dem Motto „Aufstehen FÜR ...“ konnte die IG Metall ein überparteiliches Bündnis mit SPD, CDU, Bündnis9o/Die Grünen, FDP und Freien Wählern organisieren. In diesem Bündnis wurde eine gemeinsame Erklärung verfasst, die Unterstützer suchte, sowie eine "Hockete ${ }^{\text {“ } 2}$ organisiert, an der sich weitere gesellschaftlich wichtige Organisationen beteiligten, u. a. Lebenshilfe, Naturfreunde, Rastatter Frieden, Mehr Demokratie, Junge Flüchtlinge Rastatt, Die Linke.

Die gemeinsame Erklärung fand sofort wichtige Erstunterzeichner, nämlich die Oberbürgermeister und Bürgermeister des Landkreises, Landes- und Bundestagsabgeordnete, Geschäftsführer und Werkleiter wichtiger Betriebe, kirchliche Würdenträger - und natürlich Betriebsratsvorsitzende und andere bekannte Vertreterinnen und Vertreter aus den Betrieben. Die Erklärung, die für demokratische Werte steht, wurde gesellschaftlich breit getragen. Und die Mehrheit der Menschen in der Region

1 Die unterschiedlichen Werte sind spätestens mit der Veröffentlichung des Videomitschnitts der "Compact"-Konferenz vom November 2017 (u.a. mit Björn Höcke) mehr als deutlich geworden.

2 Hockete bezeichnet im schwäbischen Sprachraum ein zwangloses, gemütliches Treffen/Beieinandersitzen zum geselligen Austausch. 
hat nicht nur die Erklärung positiv bewertet, sondern auch die Veranstaltung. Dieses sichtbare Einstehen für demokratische Werte hat nicht nur ohnehin politisch aktive Gruppierungen angezogen, sondern auch Menschen auf dem Weg von ihren samstäglichen Einkäufen. Sie blieben stehen, hörten zu, erkundigten sich an den Ständen.

Ausgesprochen erfolgreich war auch die Initiative der IG Metall Gaggenau, den Bundestagspräsidenten Dr. Norbert Lammert zu einer Veranstaltung zum Thema „Zukunft braucht Demokratie - Demokratie braucht Zukunft“ einzuladen. Dass diese lang geplante Veranstaltung nur einen Tag vor dem AfD-Landesparteitag im nahegelegenen Rastatt stattfand, war purer Zufall. Bei dieser Veranstaltung zeigte sich, wie wichtig es ist, auch über das unmittelbare gewerkschaftliche Umfeld hinaus gemeinsam mit bekannten Persönlichkeiten für demokratische Werte einzustehen. Es waren begeisterte Gewerkschafterinnen und Gewerkschafter, die die Veranstaltung verließen. Für regionale Politiker und sonstige Personen der regionalen Öffentlichkeit war es eine Ehre, zu dieser Veranstaltung eingeladen zu werden. Und es fragten natürlich auch Menschen aus der Region an, die noch nie bei einer gewerkschaftlichen Veranstaltung gewesen waren. Die regionale mediale Aufmerksamkeit war riesig. Zentrale Inhalte und Positionen der IG-Metall-Wertekampagne flossen in die sogenannte "Gaggenauer Erklärung" der IG Metall ein, deren Erstunterzeichner Norbert Lammert war, gefolgt von den anderen Teilnehmenden dieser Veranstaltung.

Es sei noch ein drittes Beispiel für das Wirken der IG Metall, für ihr Eintreten für demokratische, gewerkschaftspolitische Werte in der breiten politischen Öffentlichkeit genannt: Es gibt eine gute Tradition bei der IG Metall Gaggenau, bei überregionalen Wahlen die örtlichen Kandidatinnen und Kandidaten einzuladen, um über deren Wahlprogramme zu diskutieren - anhand der gewerkschaftlichen Anforderungen an die Politik.

Im Vorfeld der Bundestagswahl 2017 warf die Vorbereitung eines solchen Podiums mit den regionalen Kandidatinnen und Kandidaten für ein Bundestagsmandat aber besondere Diskussionsbedarfe und Herausforderungen auf: Sollen auf AfD-Listen Kandidierende eingeladen werden? Die Diskussion hierüber war außergewöhnlich kontrovers und ausdrücklich ergebnisoffen. Für und Wider, Chancen und Risiken wurden zusammengetragen und bewertet, politikwissenschaftliche Expertise zum Wahlverhalten und zu Wahlprognosen hinzugezogen, Diskussionen aus betrieblichen Gremien wurden einbezogen, um ein breites Bild aus den Betrieben der Region zu erhalten. Das alles führte zu dem Beschluss, die Kandidaten der AfD zur Podiumsdiskussion einzuladen. Voraussetzung für die Teilnahme für jede Kandidatin und jeden Kandidaten war die Unterzeichnung und Stellungnahme zur „Gaggenauer Erklärung“. Das haben alle Eingeladenen getan: SPD, CDU, Bündins9o/Die Grünen, FDP und AfD.

Im Rückblick ist festzustellen: Es kamen tatsächlich mehr und andere Interessierte als bei „üblichen“ Podiums- diskussionen vor Wahlen. Der IG Metall Gaggenau wurde es hoch angerechnet, die AfD nicht einfach auszugrenzen. Allerdings sind einige aktive Gewerkschafter der Veranstaltung aufgrund der AfD-Beteiligung auch bewusst fern geblieben. Der AfD-Kandidat selbst gab sich ausgesprochen „weichgespült“: Zu den Anforderungen der IG Metall an die Politik äußerte er seine persönliche Meinung, aber nicht die des AfD-Programms. Ob wir bei einer nächsten überregionalen Wahlveranstaltung AfD-Kandidaten erneut beteiligen, ist aus heutiger Sicht zweifelhaft. Vor einer Entscheidung wird es aber erneut einen ausführlichen Diskussionsprozess geben.

\section{Schlussfolgerungen und Ausblick}

Es wäre anmaßend und unrealistisch zu meinen, dass die vielfältigen Aktivitäten der IG Metall Gaggenau in Betrieben und Öffentlichkeit für gewerkschaftliche und demokratische Werte und gegen Ausgrenzung und Rechtspopulismus ausreichend wären, um den „Kampf um die Köpfe“ zu gewinnen. Damit stellt sich auch in innergewerkschaftlichen Diskussionen die Frage: Hat sich der Aufwand gelohnt? Die Antwort der betrieblichen Aktiven fällt eindeutig aus: Es war richtig, selbst dann, wenn sich der Ertrag (noch) nicht in Wahlergebnissen widerspiegelt. Deutlich wurde und wird allerdings, wofür die IG Metall in der Region steht.

Wenn wir feststellen, dass immer weniger Menschen bereit sind, für ihre Vorstellung von Gesellschaft und Politik auf die Straße zu gehen, dann ist die Konsequenz zu überlegen, welche anderen Formen geeignet und möglich sind, um die demokratische Mehrheit zu erreichen und diejenigen zurückzugewinnen, die sich von den einfachen Ursachenerklärungen, Verantwortungszuschreibungen und Scheinlösungen rechtspopulistischer Positionen angezogen fühlen.

In Tarifrunden ist die IG Metall in der Lage, wirklich Massen zu mobilisieren. Das bedeutet nicht, dass die gleichen Menschen sich in gleicher Weise aktiv für gesellschaftspolitische Themen engagieren. Es heißt jedoch auch nicht, dass sie zu diesen Themen die Positionen der IG Metall ablehnen. Aber die Aktions- und Kommunikationsformen müssen angepasst werden. Daran gilt es, weiter zu arbeiten.

Die Grundüberlegung, öffentlichkeitswirksam deutlich zu machen, wofür die IG Metall steht, anstatt zu betonen, wogegen sie ist, hat sich bewährt. Auch daran werden wir weiterarbeiten. Das bedeutet zugleich, dass wir uns zwar zu den von rechtspopulistischen Strömungen und Parteien öffentlichkeitswirksam platzierten Themen (wie Flucht und Asyl) deutlich positionieren werden, uns jedoch nicht darauf reduzieren lassen. Es gibt weitere wichtige betriebliche und gesellschaftliche Zukunftsthemen, die für unser gesellschaftliches Wohl und für unsere Arbeitsplätze wichtig sind (u. a. Digitalisierung, Mobilität, Transformation des Antriebsstrangs). Gestalten ist hier 
der Anspruch der IG Metall. Das muss in der alltäglichen betrieblichen Arbeit deutlich werden.

Damit allein wird rechtspopulistisches Denken nicht verschwinden. Aber unser Ansatz schärft die Sichtweise auf wichtige Zukunftsthemen. Wir müssen auch nicht auf alles Antworten haben. Fragen zu stellen und diese zu diskutieren, ist gleichfalls wichtig. Verklärte Sichtweisen helfen uns genauso wenig weiter wie einfache Lösungen. Das macht Gewerkschaftsarbeit ja auch so schwierig: Es gibt keine einfachen Lösungen. Komplexe Themen brauchen mehrschichtige und manchmal auch schwierige Lösungsansätze. Dazu stehen wir.

\section{AUTOREN}

CLAUDIA PETER ist Erste Bevollmächtigte der IG Metall Gaggenau.

@ claudia.peter@igmetall.de

MICHAEL BRECHT ist Betriebsratsvorsitzender im Mercedes-Benz-Werk Gaggenau und Gesamtbetriebsratsvorsitzender der Daimler AG.

michael.brecht@daimler.com 